Special Issue of First International Conference on Advancements in Engineering \& Technology (ICAET- 2020)

\title{
Applications of Vedic mathematics in Algebra
}

Sarita Devi ${ }^{\text {. }}$

${ }^{1}$ Department of Applied Sciences and Humanities, Pillai HOC college of Engineering and Technology, Rasayani. Raigad, Navi Mumbai, Maharashtra,, India.

sarita2009devi@gmail.com ${ }^{1}$.

\begin{abstract}
Vedic mathematics is an interesting, speedy, simple logical \& integral part of our ancient Indian culture using traditional mathematics, which finds its origin in our Vedas especially "Atharva Veda" and is mainly based on 16 principles called "Sutras" \& 13 Sub-Sutras. Applied to almost every branch of mathematics. The interesting part in Vedic mathematics is, you can mostly check your calculation and know whether you are right or wrong in few seconds and that makes it more enjoyable. Here, discuss some applications of Vedic mathematics in one of its field i.e. Algebra and see how it saves a lot of time \& efforts in solving the problems.
\end{abstract}

Keywords: Sutras, Sub-Sutras, Algebra, Atharva.

\section{Introduction}

The Vedas \& Upaveda are unlimited storehouse of knowledge, which were probed extensively by and this led to the development of the sixteen Sutras and thirteen Sub-Sutras. Therefore, developed methods \& techniques, elaborating the principles contained in these Sutras \& Sub- Sutras is called Vedic mathematics.

Sutras, which are basically single line phrases, is based on a rational way of thinking, which improves intuition, creativity and emphasizes on development of our mental abilities, which is the bottom-line of the mastery, that is seen, in mathematical geniuses of the present and the past. The Sutras are correlated. A single Sutra/Formula can be used to perform various arithmetic calculation, all the basic calculations can be done using different available methods, and it isup to the students to choose the method they find to be more comfortable. Applications of the sixteen Sutras can be found in the different branches of mathematics viz. geometry, calculus, arithmetic's, trigonometry etc. and these Sutras make all the mathematical calculations easy, fast and error free, which in turn, makes mathematics more joyful \& is a great confidence booster for students who fear mathematics. No wonder then, that, this Vedic mathematics is being adopted by various professionals, scientists, taught in some of the most prestigious institutes worldwide \& especially students preparing for various big competitions, to achieve better performance.[1-4]

\section{Vedic Sutras for solving simultaneous equation}

Vedic Sutras for solving simultaneous equation are

- Paravartya Yojayet

- Anurupye Sunyamanyat

- Sankalana Vyavakalana-bhyam 


\subsection{ParavartyaYojayet}

This method is applicable for all sorts of linear simultaneous equations. A simple idea for finding value of $\mathrm{x}$, start with $\mathrm{y}$ - coefficient and independent terms and cross multiply them in the forward direction, the sign between the two cross multiplication is minus (-). For y, start with the independent term and $\mathrm{x}$ - coefficient and cross-multiply them in the backward direction. The sign between the cross-multiplication result is minus (-). For the result of the denominator, take the coefficient of variable only and crossmultiply then in backward direction.

Suppose, have the following set of simultaneous equations: -

$\mathrm{a} 1 \mathrm{x}+\mathrm{b} 1 \mathrm{y}=\mathrm{c} 1$

$a 2 x+b 2 y=c 2$

In order to get the numerator of $\mathrm{x}$, leave the coefficient of $x$ and write the coefficient of $y$ and the independent term and cross multiply them with a minus sign in between the cross product in rightward direction as shown here:-[4-6]

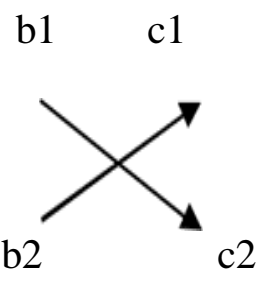

$=\mathrm{b} 1 \mathrm{c} 2-\mathrm{b} 2 \mathrm{c} 1$

Again, to get numerator of $y$, leave the coefficient of $y$ and take only the coefficient of $x$ and the independent term into consideration. As know the sutra moves in a cyclic order, so start with independent term first. Cross multiplication of the independent term and coefficient of $\mathrm{x}$ will give the numerator of $y$.

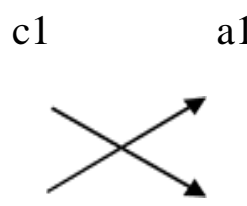

c2

$$
\text { a2 }
$$

Here is an example to understand the entire concept by this simple diagrammatical structure.

Numerator of $\mathrm{x} \quad$ Numerator of $\mathrm{y}$ Denominator

b

$\mathrm{c} 1$

a1

b1

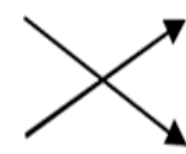

b2

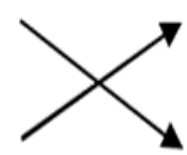

a2
Here is an example to understand this concept.

Example 1: Simplify for $x$ and $y, 3 x+4 y=10$;

$4 x-2 y=-16$

Coefficient of y Independent term

4

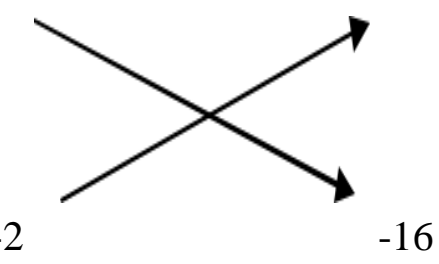

$\mathrm{x}=$

Coefficient of $x$ Coefficient of $y$

3

4

4

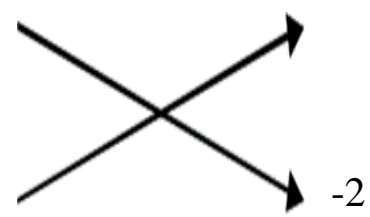




\section{Independent Term Coefficient of $\mathrm{x}$}

10
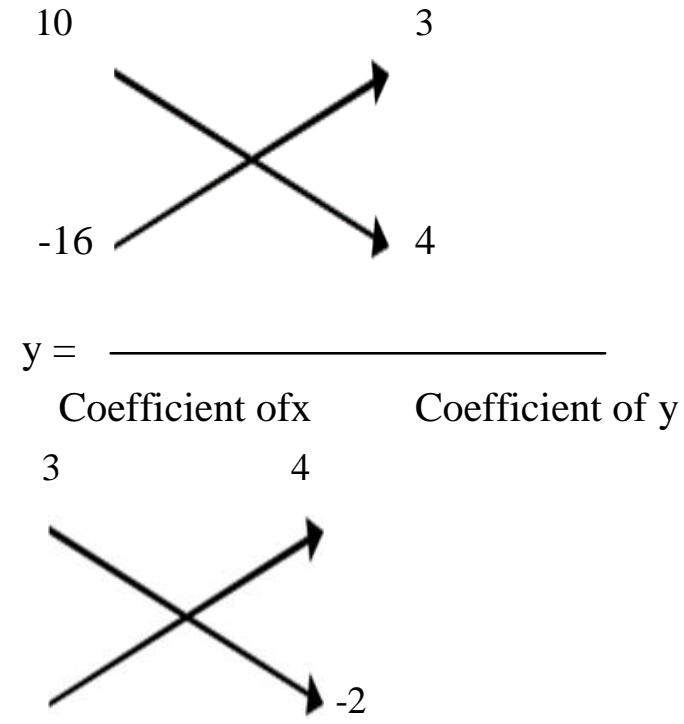

4

$\mathrm{x}=-2, \mathrm{y}=4$

\subsection{Anurupye Sunyamanyat:}

This Vedic sutra says -

If one is in ratio, the other one is zero, it means when the ratio of $\mathrm{x}$ or $\mathrm{y}$ is equal to that of independent term, put the ratio of $\mathrm{y}$ or $\mathrm{x}=0$. To understand here is an example:

Example 2: Simplify for $\mathrm{x}$ and $\mathrm{y}$

$$
\begin{aligned}
& 7 x+6 y=70 \\
& 14 x+13 y
\end{aligned}
$$

The ratio of coefficients of $\mathrm{x}$ is $1: 2$ which is equal to the ratio of the independent term. So according to above sutra, we put $\mathrm{y}=0$ in either of the equations to get the value of $x$.

For $\mathrm{y}=0, \quad 7 \mathrm{x}=70, \quad \mathrm{x}=10$

For $\mathrm{x}=10 \quad \mathrm{y}=0$, is the solution

\subsection{Anurupye Sunyamanyat:}

This sutra simply says: Addition and subtraction.

When there is coefficient of $x$ in 1 st equation is equal to the coefficient of $y$ in 2 nd equation and vice versa. This sutra works well. To understand this here is an example:

Example3: Solve for $\mathrm{x}$ andy.

$$
\begin{aligned}
& 1955 x-476 y=2482 \\
& 476 x-1955 y=-49131
\end{aligned}
$$

Adding (A) and (B), equation becomes, $2431 x-2431 y=-2431$;

$$
\Longrightarrow \mathrm{x}-\mathrm{y}=-1
$$

Subtract (B) from (A):

$$
1479 x-1479 y=7395 \text {; }
$$

$$
\Longrightarrow \mathrm{x}+\mathrm{y}=5
$$

Add (C) and (D), value of $x=2$ subtract (D) from $(C)$, value of $y=3$.

\section{Conclusion}

Therefore, while applying Vedic mathematics one can versatility in solving problems and at the same time, this helps to decide on the best method possible in solving a particular type of problem. The beauty of Vedic mathematics is in its inventiveness, which one experiences while applying. As one can see in the above methods that with good practice of the Vedic mathematics one can do time consuming complex Problems far more easily and faster.

\section{References}

[1].Jagadguru Swami Sri Bharati Krisna Tirthaji (1986), Vedic Mathematics or Sixteen Simple Sutras from the Vedas. Motilal Banarasidas, Varanasi(India).

[2].Rajesh Kumar Thakur: Advanced Vedic Mathematics.

[3].Goel, A. (2006), Learn and teach mathematics, New Delhi: Authors Press, Scholarly Books.

[4].Rajesh Kumar Thakur - The essentials of Vedic mathematics

[5].J.T. Glover - Vedic mathematics for schools Book2.

[6].http://www.vedicmaths.org. 